SHEP 96-08

UPRF-96-458

hep-th/9602156

\title{
Gauge Invariance, the Quantum Action Principle, and the Renormalization Group
}

\author{
Marco D’Attanasio ${ }^{1,2}$ and Tim R. Morris ${ }^{1}$ \\ 1. Physics Department, University of Southampton, Southampton SO17 1BJ, UK \\ 2. I.N.F.N., Gruppo collegato di Parma, viale delle Scienze, 43100 Parma, Italy
}

\begin{abstract}
If the Wilsonian renormalization group $(R G)$ is formulated with a cutoff that breaks gauge invariance, then gauge invariance may be recovered only once the cutoff is removed and only once a set of effective Ward identities is imposed. We show that an effective Quantum Action Principle can be formulated in perturbation theory which enables the effective Ward identities to be solved order by order, even if the theory requires non-vanishing subtraction points. The difficulties encountered with non-perturbative approximations are briefly discussed.
\end{abstract}




\section{Introduction}

In trying to formulate a non-perturbative RG円 for a gauge theory, we encounter the problem that the division of momenta into large or small (according to some scale $\Lambda$ ) - which is fundamental to the exact RG approach - is incompatible with gauge invariance. This is easy to appreciate if one considers a homogeneous gauge transformation $\Omega$ on some matter fields $\Phi(x)$,

$$
\Phi(x) \mapsto \Omega(x) \Phi(x)
$$

Since in momentum space, $\Phi(p)$ is mapped into a convolution with the gauge transformation, any division of momenta into high and low is seen not to be preserved by gauge transformations. In order to solve this problem, clearly we are presented with two options:

(a) We break the gauge invariance in intermediate steps, and aim to recover the gauge invariance once $\Lambda$ is removed - by imposition of some constraints.

(b) We generalize the RG in such a way that it manifestly preserves the gauge invariance.

Clearly, manifest preservation of gauge invariance would be preferable. Unfortunately for all but the very simplest case of pure U(1) gauge theory [1], we encounter in option (b) the well known problem that it is not easy to regularise non-perturbatively while preserving both Poincaré invariance and gauge invariance. Actually the problem is worse than that [1], because the resulting RG must also allow for manageable approximations, and this has so far required that the cutoff be effectively placed inside a free propagator, breaking the gauge invariance for all but pure $\mathrm{U}(1)$ gauge theory. We will not discuss this option further here, but instead give a detailed study of option (a).

Option (a) can be carried out to all orders in perturbation theory, relatively straightforwardly, since, as we will show, an analogue of the Quantum Action Principle (QAP) exists for the solution of the broken Ward identities. When these are solved, the unbroken Ward identities are guaranteed to hold once the cutoff is removed. Indeed in this way, renormalized physical Green functions, with the correct gauge dependence, evaluated at non-zero subtraction points (when necessary, i.e. when massless particles are present), may be constructed order by order in the couplings. Our study of the QAP also serves as a basis to study non-perturbative approximations. We point out the difficulties involved in finding viable non-perturbative approximations, i.e. ones in which the appropriate Ward identities are exactly obeyed.

Some comments on the use of the background field method are now necessary. If the $\mathrm{RG}$ is combined with the background field method [2, 3], then background gauge invariance may be maintained by replacing the division of momenta into high or low, by a division of eigenvalues of the background covariant Laplacian. The problems of option (b) above are not encountered here because the background field does not propagate. Equally however, note that nor does background field invariance replace the need to ensure that the quantum gauge invariance ${ }^{\mathbb{2}}$ is respected by the quantum fields, for example it is the latter that ensures that longitudinal modes are properly cancelled by ghosts in internal propagators, i.e. that

\footnotetext{
${ }^{1}$ i.e. the Wilsonian RG, also called the exact RG.

${ }^{2}$ i.e. BRS invariance in gauge fixed systems
} 
unitarity is maintained, not the former. Thus these methods must also be treated according to category (a).

Lastly, let us stress that it is crucial that approximations of the RG do not of themselves result in breaking of the (quantum) gauge invariance in the final answer. This requires, for (a), exact preservation of the broken Ward identities. Of course, otherwise, spurious violations of unitarity will be encountered, but many other properties of gauge theories would also be lost. Amongst the most important, we mention Elitzurs theorem, which in turn implies the non-existence of local order parameters [ [4], Instantons (more generally any topologically non-trivial principal fibre bundles), the existence of Gribov fundamental domains.[3 and anomalies. It is hard to see how these properties would be properly incorporated if gauge invariance is only "approximately" conserved. Indeed it may even be the case that some of these properties cannot be recovered at all by method (a). In this case, non-perturbatively, Wilson RGs of the form (a) would fail to access certain continuum limits. (Equivalently, all regularizations with gauge non-invariant physical cutoffs would fail to be in the basin of attraction of the relevant fixed points.)

Let us assume that all the modes of the fields above $\Lambda$ are integrated out to generate a Wilsonian effective action $S(\Lambda)$. The dependence on $\Lambda$ of $S(\Lambda)$ can be translated into a differential evolution equation, which in general has the form

$$
\partial_{\Lambda} S(\Lambda)=F[S(\Lambda) ; \Lambda]
$$

This flow equation can be used to define the theory. However, it is crucial to understand the symmetry properties of the effective action, i.e. of the required solution to (2). At the effective level the local gauge symmetry of the theory is expressed by a set of effective Ward identities (see sect. 4)

$$
\Delta[S(\Lambda) ; \Lambda]=0 .
$$

The functional $\Delta$ satisfies a linear evolution equation

$$
\partial_{\Lambda} \Delta=L \cdot \Delta
$$

We address the problem of the locality of $\Delta$, i.e. we discuss the so-called Quantum Action Principle (QAP) [6, 7, 8, 9], which is a major step towards (3). The QAP will be proven in perturbation theory for a general gauge theory, no matter if the theory contains massless particles which require subtraction points to define the local approximants of $\Delta$ and $S$. We will find, after Legendre transforming, that

$$
L=\hbar L_{0}+\mathcal{O}\left(\hbar^{2}\right)
$$

From this, it follows that $\partial_{\Lambda} \Delta^{(\ell)}=0$, where $\ell$ is the first loop order in which $\Delta$ is nonvanishing. Then it will be enough to show that $\Delta^{(\ell)}$ is local at a particular value of $\Lambda$ to get the QAP in perturbation theory.

The perturbative QAP is a well-known property in field theory. However, it is interesting to discuss it with the modern point of view of Wilson RG method. Moreover there have been attempts to give eq. (2) a meaning which goes beyond perturbation theory (for instance by performing truncations of the effective action). Eq. (4) can be used to study how the

\footnotetext{
${ }^{3}$ yielding non-perturbative corrections to the standard Ward identities [5]
} 
evolution of the couplings "deviates" from the gauge invariant trajectory given by (2) and (3).

In the next section we recall the QAP. In sect. 3 the RG flow equations are constructed and discussed, and finally in sect. 4, the effective Ward identities are derived and we prove that they may be cast as local in each order of perturbation theory. The final section concludes with some remarks on non-perturbative approximations, in particular on the use of a derivative expansion, and/or truncations [1, 2, 3, 10, 11, 12]. Apart from these final remarks, we will work in a perturbative language, however the equations (2)-(4) nevertheless will easily be seen to be valid non-perturbatively.

\section{The Quantum Action Principle}

The Quantum Action Principle describes the response of a quantum field theory under a field transformation. Thus it is a fundamental tool in the construction of field theories with symmetry properties. Let us consider an infinitesimal continuous transformation of the fields $\Phi$ of the theory

$$
\delta \Phi_{i}(x)=\epsilon(x) P_{i}[\Phi(x)],
$$

where the $P_{i}[\Phi]$ are (anticommuting) polynomials in the fields, corresponding in the case of gauge theories to BRS transformations, and $\epsilon$ is an anticommuting parameter. The path integral representation of the Euclidean generating functional is

$$
Z[J, \eta]=\int \mathcal{D} \Phi e^{-S[\Phi, \eta]+J_{i} \Phi_{i}}
$$

where (perturbatively)

$$
S[\Phi, \eta]=S_{\text {classical }}[\Phi]+S_{\text {counterterms }}[\Phi]-\eta_{i} P_{i}[\Phi]
$$

and a regulator of ultraviolet (UV) divergences is assumed. By performing the field transformation with a constant $\epsilon$ we get the identity

$$
\int d^{4} x J_{i}(x) \frac{\delta Z[J, \eta]}{\delta \eta_{i}(x)}=\int \mathcal{D} \Phi \Delta[\Phi, \eta] e^{-S[\Phi, \eta]+J_{i} \Phi_{i}}
$$

where

$$
\Delta[\Phi, \eta]=\int d^{4} x \frac{\delta^{2} S(\Phi, \eta)}{\delta \Phi_{i}(x) \delta \eta_{i}(x)}-\int d^{4} x \frac{\delta S[\Phi, \eta]}{\delta \Phi_{i}(x)} \frac{\delta S[\Phi, \eta]}{\delta \eta_{i}(x)} \equiv \mathcal{B}_{\Phi} \cdot S .
$$

Then the response of the system is given by the insertion of a local operator of dimension 4 minus the dimension of the field $\Phi_{i}$ plus the dimension of the corresponding variation $P_{i}[\Phi]$. When removing the regulator (UV limit) the l.h.s. is finite, at least in perturbation theory, and this ensures that also the insertion of the operator $\Delta$ is finite in the UV limit. In terms of the generating functional of 1PI Green functions, eq. (8) reads

$$
\int d^{4} x \frac{\delta \Gamma[\Phi, \eta]}{\delta \Phi_{i}(x)} \frac{\delta \Gamma[\Phi, \eta]}{\delta \eta_{i}(x)}=[\Delta \Gamma]
$$

where $\Gamma[\Phi, \eta]=-\log Z[J, \eta]+J_{i} \Phi_{i}$ and the r.h.s. stands for the 1 PI functions with the insertion of $\Delta$. 
In general one is interested in solving the equation $\Delta=0$. Notice that in perturbation theory $[\Delta \Gamma]=\Delta+\mathcal{O}(\hbar)$, and from this it follows that the insertion of $\Delta$ is local at the first order in which $\Delta$ itself is non-vanishing. Since there are only a finite number of local operators of the correct dimension, it follows that the equation $\Delta=0$ is, order by order, a finite number of conditions, which can eventually be satisfied by fine-tuning [13 the parameters in the action (7). The relations (8) and (10), together with locality of $\Delta$ as just described, is known as the QAP.

We want to generalize these concepts to an effective theory, obtained from a more fundamental one after integrating the high energy degrees of freedom. It is clear that such a procedure of integration will generate effective non-locall interactions and also the field transformations will become non-local. Thus it is not evident how the QAP can be obtained at the effective level.

\section{RG flow equations}

The main idea of Wilson RG [14, 15, 16, 17] is to consider an interacting field theory as an effective theory, that is to regard the high frequency modes of the fields of the theory as generating effective couplings for the low energy modes. In this picture one introduces an UV cutoff $\Lambda_{0}$, which is a just a tool to define the Green functions of the theory. For simplicity we will consider local interactions. Then a scale $\Lambda$ is introduced and the frequencies between $\Lambda$ and $\Lambda_{0}$ are viewed as generating interactions for the frequencies lower than $\Lambda$. The physical parameters are fixed at a physical scale $\Lambda_{R} \leq \Lambda$. In particular we will choose $\Lambda_{R}=0$.

We will denote with $\Phi_{a}=\{\phi, \psi, \bar{\psi}\}$ the fields of the theory (the $\phi$ 's are commuting fields while the $\bar{\psi}, \psi$ are anticommuting, fermions or ghosts) and $J_{a}=\{j, \bar{\chi},-\chi\}$ the corresponding sources. As in the previous section, the sources $\eta$ are coupled to the composite operators defining the symmetry transformations of the fields. Summations over internal indices are understood. The free propagators are collected in the matrix

$$
D_{a b}^{-1}=\left(\begin{array}{ccc}
D_{1}^{-1} & 0 & 0 \\
0 & 0 & -D_{2}^{-1} \\
0 & D_{2}^{-1} & 0
\end{array}\right)
$$

where $D_{1}$ and $D_{2}$ are the free propagators of the $\phi$ and $\psi, \bar{\psi}$ respectively. The Euclidean generating functional is

$$
Z[J, \eta]=\int \mathcal{D} \Phi \exp \left\{-\frac{1}{2}\left(\Phi, D^{-1} \Phi\right)_{0 \Lambda_{0}}-S_{\mathrm{int}}\left[\Phi, \eta ; \Lambda_{0}\right]+(J, \Phi)_{0 \Lambda_{0}}\right\}
$$

where the interaction action $S_{\text {int }}$ contains local, renormalizable interactions and the sources for the variations of the fields, as in (77). We have introduced a cutoff scalar product

$$
(f, g)_{\Lambda \Lambda_{0}} \equiv \int_{p} K_{\Lambda \Lambda_{0}}^{-1}(p) f_{a}(-p) g_{a}(p), \quad \int_{p} \equiv \int \frac{d^{4} p}{(2 \pi)^{4}},
$$

\footnotetext{
${ }^{4}$ by "non-local" we mean here a series of local interactions of arbitrarily high numbers of derivatives. These interactions are sometimes referred as quasi-local.
} 
where $K_{\Lambda \Lambda_{0}}(p)$ is a cutoff function which is one for $\Lambda^{2} \leq p^{2} \leq \Lambda_{0}^{2}$ and rapidly vanishing outside this interval. In order to be rigorous, this function can be taken to be always different from zero and of class $C^{\infty}$ [15]. By integrating over the high energy modes one finds

$$
Z[J, \eta]=N\left[J ; \Lambda, \Lambda_{0}\right] \int \mathcal{D} \Phi \exp \left\{-\frac{1}{2}\left(\Phi, D^{-1} \Phi\right)_{0 \Lambda}-S_{\text {eff }}\left[\Phi, \eta ; \Lambda, \Lambda_{0}\right]+(J, \Phi)_{0 \Lambda}\right\},
$$

where the coefficient $N$ is given by

$$
\log N\left[J ; \Lambda, \Lambda_{0}\right]=\frac{1}{2}(J, D J)_{0 \Lambda_{0}}-\frac{1}{2}(J, D J)_{0 \Lambda} .
$$

The functional $S_{\text {eff }}$ is the Wilsonian effective action and contains the effective interaction coming from the frequencies $p^{2}>\Lambda^{2}$. It is possible to show that this functional is equivalent to a generalization of (12), in which the free propagators contain $\Lambda$ as an infrared cutoff [19, 20]. We thus define the generating functional of the cutoff connected Green function

$$
e^{-W\left[J, \eta ; \Lambda, \Lambda_{0}\right]}=\int \mathcal{D} \Phi \exp \left\{-\frac{1}{2}\left(\Phi, D^{-1} \Phi\right)_{\Lambda \Lambda_{0}}-S_{\text {int }}\left[\Phi, \eta ; \Lambda_{0}\right]+\int_{p} J \Phi\right\} .
$$

Then we have

$$
S_{\mathrm{eff}}\left[\Phi, \eta ; \Lambda, \Lambda_{0}\right]-\frac{1}{2}\left(\Phi, D^{-1} \Phi\right)_{\Lambda \Lambda_{0}}=W\left[K_{\Lambda \Lambda_{0}}^{-1} J^{\prime}, \eta ; \Lambda, \Lambda_{0}\right]
$$

where we introduced the useful source

$$
J_{a}^{\prime}=D_{a b}^{-1} \Phi_{b}
$$

Namely, apart for the tree level two point functions, the Wilsonian effective action is the generating functional of the connected amputated cutoff Green function. As one expects, it is technically easier to study the Legendre transform of $W\left[J, \eta ; \Lambda, \Lambda_{0}\right]$, which we call "cutoff effective action" and is a generalization of the usual quantum effective action, since it contains the infrared cutoff $\Lambda$ in the free propagators [18, 19, 20]

$$
\Gamma\left[\Phi, \eta ; \Lambda, \Lambda_{0}\right]=W\left[J, \eta ; \Lambda, \Lambda_{0}\right]+\int_{p} J \Phi .
$$

In the limits $\Lambda \rightarrow 0$ and $\Lambda_{0} \rightarrow \infty$, one recovers the physical quantum effective action. Both these limits can be taken in perturbation theory [15, 17, 19, 21, 22, 23]. In particular the dependence on the ultraviolet cutoff $\Lambda_{0}$ will be sometimes understood.

We now come to the discussion of the $\Lambda$-dependence of the Wilsonian and cutoff effective actions. By derivating the corresponding definitions with respect to $\Lambda$ one finds the following flow equations in $\Lambda$, the Wilson or "exact" renormalization group equations.

$$
\begin{aligned}
& \Lambda \partial_{\Lambda} S_{\mathrm{eff}}\left[\Phi, \eta ; \Lambda, \Lambda_{0}\right]=-e^{S_{\mathrm{eff}}}\left[\frac{1}{2} \Lambda \partial_{\Lambda}\left(K_{\Lambda \Lambda_{0}} \frac{\delta}{\delta \Phi}, K_{\Lambda \Lambda_{0}} D \frac{\delta}{\delta \Phi}\right)_{\Lambda \Lambda_{0}}\right] e^{-S_{\mathrm{eff}}} \\
& \Lambda \partial_{\Lambda} \Pi\left[\Phi, \eta ; \Lambda, \Lambda_{0}\right]=-\frac{1}{2} \int_{q}\left[\Lambda \partial_{\Lambda} K_{\Lambda \Lambda_{0}}^{-1}(q)\right](-1)^{\delta_{a}} D_{a b}^{-1}(q)\left(\frac{\delta^{2} \Gamma}{\delta \Phi_{a}(q) \delta \Phi_{b}(-q)}\right)^{-1},
\end{aligned}
$$

where $\delta_{a}=1$ if $\Phi_{a}$ is a fermionic field and 0 otherwise and

$$
\Pi=\Gamma-\frac{1}{2}\left(\Phi, D^{-1} \Phi\right)_{\Lambda \Lambda_{0}}+\frac{1}{2}\left(\Phi, D^{-1} \Phi\right)_{0 \Lambda_{0}}
$$


is the cutoff effective action in which the infrared cutoff in the free propagators has been removed. In eq. (19) the inverse of the second derivative of $\Gamma$ is the matrix inverse taken in the space of indices $a, b$. (N.B. Functional derivatives are defined with respect to the measure thus $\frac{\delta}{\delta J(q)} \int_{p} J \Phi=\Phi(q)$, etc.)

We see an essential feature: the flow equation for $S_{\text {eff }}$ contains in the r.h.s. terms of the same loop order as the 1.h.s.. Thus in order to perform any perturbative study a filtration [24 (i.e. the introduction of a field-counting operator) in the space of vertices is required and the analysis at any loop order must be done by starting from the vertices with lower number of external fields. However, these terms are 1-particle-reducible, so they disappear in the flow equation for the cutoff effective action, thus rendering the latter preferable in perturbation theory.

In order to integrate the RG equations (18)-(19) one has to supply the boundary conditions. For this reason it is useful to split the cutoff effective action into two parts. One performs a Taylor expansion of the cutoff vertices around vanishing momenta. If there are massless fields, the expansion must be done around non-vanishing subtraction points. This expansion will have coefficients of decreasing dimension. These coefficients are the couplings of the theory. The "relevant" part is obtained by keeping the terms with coefficients having non-negative dimension (relevant couplings). All the remaining part is called "irrelevant". For instance in the scalar case one gets for the relevant part of the cutoff effective action

$$
\Pi_{\mathrm{rel}}[\phi ; \Lambda]=\frac{1}{2} \int d^{4} x \phi(x)\left[\sigma_{1}(\Lambda)+\sigma_{2}(\Lambda) \partial^{2}\right] \phi(x)+\frac{\sigma_{3}(\Lambda)}{4 !} \int d^{4} x \phi^{4}(x) .
$$

Since we expect the theory to be renormalizable, for $\Lambda \sim \Lambda_{0}$ the dimension of the irrelevant couplings should be given only by powers of $\Lambda_{0}$. Thus the simplest boundary condition for the irrelevant part of the cutoff effective action is

$$
\Gamma_{\mathrm{irr}}\left[\Phi, \eta ; \Lambda=\Lambda_{0}\right]=0
$$

Clearly, by using the boundary condition (21), there is no hope that the cutoff effective action $\Gamma\left[\Phi, \eta ; \Lambda, \Lambda_{0}\right]$ will satisfy the QAP: Since the cutoff $\Lambda_{0}$ breaks the gauge symmetry, non-local symmetry breaking terms, proportional to inverse powers of $\Lambda_{0}$, will be generated in perturbation theory by the loop corrections. Then a tremendous fine-tuning of the irrelevant vertices in $\Gamma_{\text {irr }}\left[\Phi, \eta ; \Lambda=\Lambda_{0}\right]$ is needed in order to cancel these non-invariant contributions.

Therefore with the boundary condition (21) the cutoff effective action will fulfil the QAP only in the limit $\Lambda_{0} \rightarrow \infty$. The result of the RG method is a non-local effective action $\Gamma\left[\Phi, \eta ; \Lambda, \Lambda_{0} \rightarrow \infty\right]$. In this sense, the introduction of the UV cutoff $\Lambda_{0}$ and the "unphysical" condition (21) on the effective action at the scale $\Lambda_{0}$ are only technical tools to define the theory, while the goal of the procedure is the "physical" perturbative effective action at any scale $\Lambda$.

For the relevant part it is useful to put the boundary conditions in the infrared, when most of the degrees of freedom have been integrated out (in particular at the point $\Lambda=0$, where the cutoff effective action becomes the physical one, so that the relevant couplings are related to measurable quantities). In the usual field theory language this means giving 
the physical renormalization conditions. In the language of the Wilson RG, this means that the flow in the infrared is controlled by the relevant couplings. This is a highly non-trivial step of the procedure, since at this point one really defines the theory, with its symmetries and physical couplings and masses.

We are now able to study the symmetry properties of the Wilsonian effective action and cutoff effective action. Our aim is to implement the relation

$$
\mathcal{S}_{J} \cdot Z[J, \eta] \equiv \int d^{4} x\left[j(x) \frac{\delta}{\delta \eta_{1}(x)}+\chi(x) \frac{\delta}{\delta \eta_{2}(x)}-\bar{\chi}(x) \frac{\delta}{\delta \eta_{3}(x)}\right] Z[J, \eta]=0
$$

known as Slavnov-Taylor identities. This will be done in the next section.

\section{Effective Ward identities and locality}

We perform the following cutoff change of variables in the generating functional (13) [17, 25

$$
\delta \Phi_{a}(p)=-\epsilon K_{0 \Lambda}(p) \frac{\delta S_{\mathrm{eff}}}{\delta \eta_{a}(-p)},
$$

where $\epsilon$ is a Grassmann parameter. We get the identity

$$
\begin{aligned}
& \mathcal{S}_{J} \cdot Z[J, \eta]=N\left[J ; \Lambda, \Lambda_{0}\right] \\
& \quad \times \int \mathcal{D} \Phi \Delta_{\mathrm{eff}} \exp \left\{-\frac{1}{2}\left(\Phi, D^{-1} \Phi\right)_{0 \Lambda}-S_{\mathrm{eff}}\left[\Phi, \eta ; \Lambda, \Lambda_{0}\right]+(J, \Phi)_{0 \Lambda}\right\},
\end{aligned}
$$

where the operator giving the Ward identity violation at the effective level is

$$
\Delta_{\mathrm{eff}}=-\mathcal{S}_{J^{\prime}} \cdot S_{\mathrm{eff}}+\int d^{4} p K_{0 \Lambda}(p) \mathcal{B}_{\Phi} \cdot S_{\mathrm{eff}}
$$

where $\mathcal{S}, J^{\prime}$ and $\mathcal{B}$ have been defined in (22), (16) and (9), respectively. This formula is completely analogous to (9). We see that the first term gives the usual Ward identities, while the second is coming from the low momentum modes which still have to be integrated out. In order to get information about $\Delta_{\text {eff }}$, in the following we will study in detail the properties of the flow of this operator. $\Delta_{\text {eff }}$ satisfies a linear evolution equation (found by explicit derivation) [17, 25]

$$
\begin{aligned}
\Lambda \partial_{\Lambda} \Delta_{\mathrm{eff}} & =e^{2 S_{\mathrm{eff}}}\left[\frac{1}{2} \Lambda \partial_{\Lambda}\left(K_{0 \Lambda} \frac{\delta}{\delta \Phi}, K_{0 \Lambda} D \frac{\delta \Delta_{\mathrm{eff}}}{\delta \Phi}\right)_{0 \Lambda}\right] e^{-2 S_{\mathrm{eff}}} \\
& =\int_{p}\left[\Lambda \partial_{\Lambda} K_{0 \Lambda}(p)\right]\left\{L_{1}+\hbar L_{2}\right\} \Delta_{\mathrm{eff}}
\end{aligned}
$$

where the linear operators $L_{1}$ and $L_{2}$ are given by

$$
\begin{aligned}
L_{1} & =D_{1}(p) \frac{\delta S_{\mathrm{eff}}}{\delta \phi(-p)} \frac{\delta}{\delta \phi(p)}-D_{2}(p) \frac{\delta S_{\mathrm{eff}}}{\delta \psi(-p)} \frac{\delta}{\delta \bar{\psi}(p)}+D_{2}(p) \frac{\delta S_{\mathrm{eff}}}{\delta \bar{\psi}(-p)} \frac{\delta}{\delta \psi(p)} \\
L_{2} & =-\frac{1}{2} D_{1}(p) \frac{\delta^{2}}{\delta \phi(-p) \delta \phi(p)}-D_{2}(p) \frac{\delta^{2}}{\delta \psi(-p) \delta \bar{\psi}(p)} .
\end{aligned}
$$


In eq. (24) we restored the powers of $\hbar$ in order to show how in the r.h.s. of the flow equation for $\Delta_{\text {eff }}$ there are terms at the same loop order of the l.h.s..

Since $\Delta_{\text {eff }}$ satisfies a linear equation, the gauge symmetry condition $\Delta_{\text {eff }}=0$ is verified for any $\Lambda$ if we can set to zero the boundary conditions of (24). The main point is to fix

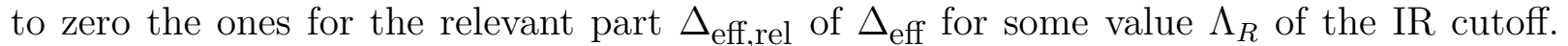
Normally $\Delta_{\text {eff,rel }}\left(\Lambda_{R}\right)=0$ is a set of constraints which overdeterminates the couplings in $S_{\text {eff }}\left(\Lambda_{R}\right)$. The number of independent constraints can be reduced by exploiting the so-called consistency conditions, which are a set of algebraic identities coming from the anticommutativity of the differential operator $\frac{\delta}{\delta \eta} \frac{\delta}{\delta \Phi}$ [17]. However, for this it is crucial the way in which the relevant parts are defined. If we keep the IR cutoff $\Lambda_{R} \neq 0$, we can extract $\Delta_{\text {eff,rel }}\left(\Lambda_{R}\right)$ by expanding the vertices of $\Delta_{\text {eff }}\left(\Lambda_{R}\right)$ around vanishing momenta, even though we are considering massless particles. The result is that the consistency conditions constrain some couplings in $\Delta_{\text {eff,rel }}\left(\Lambda_{R}\right)$, so that the set $\Delta_{\text {eff,rel }}\left(\Lambda_{R}\right)=0$ can be fulfilled

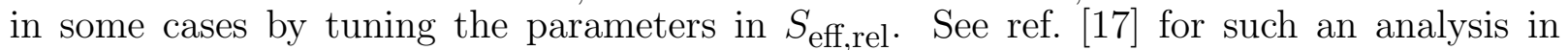
the pure gauge $\mathrm{SU}(2)$ model. If we are interested in fixing the boundary conditions at the physical point $\Lambda_{R}=0$ in a theory with one or more massless particles, we have to introduce non-vanishing subtraction points in order to define $\Delta_{\text {eff.rel }}\left(\Lambda_{R}=0\right)$. This fact could spoil the power of the consistency conditions since they now involve also irrelevant vertices of $\Delta_{\text {eff }}(0)$ evaluated at the subtraction points [25]. Thus it seems that a case-by-case analysis based on a filtration of $\Delta_{\text {eff }}$ is required in order to prove the locality of $\Delta_{\text {eff }}$ so as to restore the usefulness of the consistency conditions. This was done in ref. [25].

However these nasty irrelevant contributions are of the reducible type 25] (see the form of $L_{1}$ in (25)), and we expect they will disappear when taking the Legendre transform, similarly to what happened in passing from the Wilsonian effective action to the cutoff effective action.

From (15) and (23), the expression of $\Delta_{\text {eff }}$ in terms of $W\left[J, \eta ; \Lambda, \Lambda_{0}\right]$ is

$$
\Delta_{\mathrm{eff}}=-\mathcal{S}_{K_{0 \Lambda_{0}} J} \cdot W\left[J, \eta ; \Lambda, \Lambda_{0}\right]+\int d^{4} p \frac{K_{0 \Lambda}(p)}{K_{\Lambda \Lambda_{0}}(p)} \mathcal{B}_{J^{\prime \prime}} \cdot W\left[J, \eta ; \Lambda, \Lambda_{0}\right],
$$

where $J^{\prime \prime}=D J$. By performing the Legendre transform (17) one gets the cutoff Ward identities [12] $\left(\Delta_{\Gamma}\right.$ is $\Delta_{\text {eff }}$ expressed in terms of $\left.\Phi\right)$ :

$$
\begin{aligned}
\Delta_{\Gamma} & =-\int d^{4} p K_{0 \Lambda_{0}}(p) \frac{\delta \Gamma}{\delta \Phi_{a}(-p)} \frac{\delta \Gamma}{\delta \eta_{a}(p)}+\int_{p} \frac{K_{0 \Lambda}(p)}{K_{\Lambda \Lambda_{0}}(p)}(-1)^{\delta_{a}} D_{a b}^{-1}(p) \\
& \times\left[\Phi_{a}(p) \frac{\delta \Gamma}{\delta \eta_{b}(p)}+\int_{q}\left(\frac{\delta^{2} \Gamma}{\delta \Phi_{a}(p) \delta \Phi_{c}(q)}\right)^{-1} \frac{\delta^{2} \Gamma}{\delta \Phi_{c}(-q) \delta \eta_{b}(-p)}\right] .
\end{aligned}
$$

The expression of $\Delta_{\Gamma}$ is simpler in terms of the functional $\Pi$, defined in (20). One finds

$$
\begin{aligned}
\Delta_{\Gamma} & =-\int d^{4} p K_{0 \Lambda_{0}}(p) \frac{\delta \Pi}{\delta \Phi_{a}(-p)} \frac{\delta \Pi}{\delta \eta_{a}(p)} \\
& +\int_{p, q} \frac{K_{0 \Lambda}(p)}{K_{\Lambda \Lambda_{0}}(p)}(-1)^{\delta_{a}} D_{a b}^{-1}(p)\left(\frac{\delta^{2} \Gamma}{\delta \Phi_{a}(p) \delta \Phi_{c}(q)}\right)^{-1} \frac{\delta^{2} \Pi}{\delta \Phi_{c}(-q) \delta \eta_{b}(-p)} .
\end{aligned}
$$

Again the flow equation for the cutoff ST is found by explicit derivation. We have

$$
\Lambda \partial_{\Lambda} \Delta_{\Gamma}=\int_{p, q, r}\left[\Lambda \partial_{\Lambda} K_{\Lambda \Lambda_{0}}^{-1}(p)\right](-1)^{\delta_{d}} D_{a b}^{(-1)}(p)
$$




$$
\times \frac{1}{2}\left(\frac{\delta^{2} \Gamma}{\delta \Phi_{a}(p) \delta \Phi_{c}(q)}\right)^{-1} \frac{\delta^{2} \Delta_{\Gamma}}{\delta \Phi_{c}(-q) \delta \Phi_{d}(r)}\left(\frac{\delta^{2} \Gamma}{\delta \Phi_{d}(-r) \delta \Phi_{b}(-p)}\right)^{-1}
$$

We see how this equation has the desired property, namely the evolution of the vertices of $\Delta_{\Gamma}$ depends on vertices of $\Delta_{\Gamma}$ itself at lower loop order with respect to the l.h.s..

We now come to the discussion of the locality of $\Delta_{\Gamma}$ in perturbation theory, which is an immediate consequence of eq. (29).

If one assumes that $\Delta_{\Gamma}^{\left(\ell^{\prime}\right)}$ is vanishing at any loop order $\ell^{\prime}<\ell$, then at loop $\ell \Delta_{\Gamma}^{(\ell)}$ is $\Lambda$-independent, since the flow equation (29) becomes

$$
\Lambda \partial_{\Lambda} \Delta_{\Gamma}^{(\ell)}=0
$$

Then we choose to discuss the locality of $\Delta_{\Gamma}^{(\ell)}$ (given in $(28)$ ) at the point $\Lambda=\Lambda_{0}$. At this point the functionals $\Gamma$ and $\Pi$ are local, as follows from (21), and the free propagators vanish, so that also from $\left(\frac{\delta^{2} \Gamma}{\delta \Phi_{i}(p) \delta \Phi_{j}(q)}\right)^{-1}$ only local terms are coming. Thus the only possible source of non-local contributions is the cutoff function $K_{0 \Lambda_{0}}(p)$. It is then sufficient to take the UV limit $\Lambda_{0} \rightarrow \infty$, in which $K_{0 \Lambda_{0}} \rightarrow 1$, to get a local violation $\Delta_{\Gamma}^{(\ell)}$.

Once the locality of $\Delta_{\Gamma}^{(\ell)}$ is proven, powerful cohomological methods [17] can be used to translate the finite set of equations $\Delta_{\Gamma}^{(\ell)}=0$ into a solvable set of fine-tuning conditions on the couplings of $\Pi_{\text {rel }}^{(\ell)}$.

\section{$5 \quad$ Final remarks}

Now consider non-perturbative approximations to the flow equations. For the reasons stated in the introduction, we do not accept approximations which destroy gauge invariance. This means that eqn. (24) or equivalently (29) must be absolutely respected. The problem is that even if $S_{\text {eff }}$ and thus $\Delta_{\text {eff }}$ (or, $\Gamma$ and thus $\Delta_{\Gamma}$ ) are polynomial in the fields and/or polynomial in momenta at the cutoff scale $\Lambda_{0}$, this is not preserved by the flows (24) [respec. (29)]. Thus any succesful approximation of the flow equations would have to involve a nonpolynomial non-local action - ruling out in particular, any straightforward truncation. The underlying difficulty is that the effective BRS transformation(s) (i.e. the dependence on $\eta$ ) is also non-polynomial and non-local. In principle, knowledge of this effective transformation would help determine an appropriate form of approximations to the effective action, but of course the form of the effective BRS transformation is also unkown. By differentiating (23) with respect to $\Phi$ and $\eta$ (and recalling that $\frac{\delta}{\delta \eta} \frac{\delta}{\delta \Phi}$ is Grassmann) a set of effective Wess-Zumino consistency conditions may be derived which constrain the dependence on $\eta$. However these are no easier to solve directly than the flow equations. We conclude that the loss of the locality of the QAP in the non-perturbative domain, causes severe difficulties for all approximations that use method (a).

In spite of these conceptual difficulties in studying a truncation of the effective action, we should mention the approach followed by the authors in ref. [12. They accept the unavoidable violation to the Ward identities caused by a given truncation of the effective 
action. However, they use the flow equation for the (truncated) effective Ward identity operator $\Delta_{\text {eff }}$ to study numerically the deviation of such an approximated effective action from the gauge invariant trajectory. In this way they obtain a check of the consistency of the given truncations.

We are grateful for discussion to M. Asorey, C. Becchi, M. Bonini, U. Ellwanger, M. Hirsch, G. Marchesini and A. Weber.

\section{References}

[1] T.R. Morris, Phys. Lett. B357 (1995) 225.

[2] M. Reuter and C. Wetterich, Nucl. Phys. B417, (1994) 181.

[3] F. Freire and C. Wetterich, The Ward identity from the background field dependence of the effective action, hep-th/9601081; for a review on the background field approach see M. Reuter, Effective average actions and nonperturbative evolution equations, hep-th/9602012.

[4] See for example, C. Itzykson and J-M Drouffe, Vol 1, Statistical field theory, (1989) C.U.P.

[5] M. Asorey and F. Falceto, Ann. Phys. 196 (1989) 209; K. Fujikawa, BRST symmetric formulation of a theory with Gribov-type copies, hep-th/9510111.

[6] J.H. Lowenstein, Comm. Math. Phys. 24 (1971) 1; Y.M.P. Lam, Phys. Rev. D6 (1971) 2145 and Phys. Rev D7 (1973) 2943; T.E. Clark and J.H. Lowenstein, Nucl. Phys. B113 (1976) 109.

[7] P. Breitenlohner and D. Maison, Comm. Math. Phys. 52 (1977) 11, 39 and 55.

[8] O. Piguet and A. Rouet, Phys. Rep. 76 (1981) 1.

[9] C.M. Becchi, Lectures on the renormalization of gauge theories, in Relativity, groups and topology II (Les Houches 1983), Eds. B.S. De Witt and R. Stora (Elsevier Science Pub. 1984).

[10] T.R. Morris, Nucl. Phys. B458[FS] (1996) 477.

[11] T.R. Morris, in Lattice '94, Nucl. Phys. B(Proc. Suppl.)42 (1995) 811.

[12] U. Ellwanger, Phys. Lett. B335 (1994) 364; U. Ellwanger, M. Hirsch and A. Weber, Flow equations for the relevant part of the pure Yang-Mills action, hep-th/9506019.

[13] C.M. Becchi, A. Rouet e R. Stora, Ann. Phys. (NY) 98 (1976) 287; G. Costa, J. Julve, T. Marinucci and M. Tonin, Il Nuovo Cim. 38A (1977) 373; G. Bandelloni, C.M. Becchi, A. Blasi and R. Collina, Ann. Inst. H. Poincaré XXVIII (1978) 522; Comm. Math. Phys. 72 (1980) 239; for a review see L. Baulieu, Phys. Rep. 129 (1985) 1. 
[14] K.G. Wilson, Phys. Rev. B4 (1971) 3174,3184; F.J. Wegner and A. Houghton, Phys. Rev. A8 (1973) 401; K.G. Wilson and J.G. Kogut, Phys. Rep. 12 (1974) 75.

[15] J. Polchinski, Nucl. Phys. B231 (1984) 269.

[16] G. Gallavotti, Rev. Mod. Phys. 57 (1985) 471.

[17] C.M. Becchi, On the construction of renormalized quantum field theory using renormalization group techniques, in Elementary particles, Field theory and Statistical mechanics, Eds. M. Bonini, G. Marchesini and E. Onofri, Parma University 1993.

[18] C. Wetterich, Phys. Lett. B301 (1990) 93 and Nucl. Phys. B352 (1991) 529.

[19] M. Bonini, M. D’Attanasio and G. Marchesini, Nucl. Phys. B409 (1993) 441.

[20] T.R. Morris, Int. J. Mod. Phys. A9 (1994) 2411.

[21] P.K. Mitter and T.R. Ramadas, Comm. Math. Phys. 122 (1989) 575.

[22] R.D. Ball and R.S. Thorne, Ann. of Phys. 236 (1994) 117.

[23] M. Bonini, M. D'Attanasio and G. Marchesini, Nucl. Phys. B418 (1994) 81 and Nucl. Phys. B421 (1994) 429.

[24] C.M. Becchi, A. Blasi, G. Bonneau, R. Collina and F. Delduc, Comm. Math. Phys. $120(1988) 121$.

[25] M. Bonini, M. D'Attanasio and G. Marchesini, Nucl. Phys. B437 (1995) 163 and Phys. Lett. B346 (1995) 87; M. Bonini and M. D'Attanasio, Spontaneous symmetry breaking with Wilson renormalization group, hep-th/9507098. 